\title{
Model transformations in the UPES/UPSoC development process for embedded systems
}

\author{
Elvinia Riccobene - Patrizia Scandurra
}

Received: date / Accepted: date

\begin{abstract}
Model Based Development (MBD) aims at combining modeling languages with model transformers and code generators. Modeling languages, like profiles of the Unified Modeling Language (UML), are increasingly being adopted for specific domains of interest to alleviate the complexity of platforms and express domain concepts effectively. Moreover, system development processes based on automatic model transformations are widely required to improve the productivity and the quality of the developed systems. In this paper, we show how MBD principles and automatic model transformations provide the basis for the development process UPES and its sub-process UPSoC. They have been defined to foster in a systematic and seamless manner a model-based design methodology based on the UML2 and on UML profiles for the C/SystemC programming languages, which we developed to improve the current industrial system design flow in the Embedded Systems and System-on-Chip area.
\end{abstract}

Keywords Model Based Development (MBD) · Model transformations · Unified Modeling Language (UML) . SystemC · Embedded system design

This work is supported in part by the project Model-driven methodologies and techniques for embedded systems design and analysis based on UML, Abstract State Machines, and SystemC at STMicroelectronics, AST R\&I of Agrate Brianza (MI), Italy.

Elvinia Riccobene

Università di Milano, Dip. di Tecnologie dell'Informazione, Italy Tel.: +39-02-50330055

Fax: $+39-02-50330010$

E-mail: riccobene@dti.unimi.it

Patrizia Scandurra

Università di Milano, Dip. di Tecnologie dell'Informazione, Italy

Tel.: +39-02-50330025

Fax: $+39-02-50330010$

E-mail: scandurra@dti.unimi.it

\section{Introduction}

Model Based Development (MBD) is an emerging paradigm to software production that combines domainspecific modeling languages (DSMLs) with model transformers, analyzers, and generators. Metamodel-based modeling languages, like profiles or extensions of the OMG standard Unified Modeling Language (UML), are increasingly being defined and adopted for specific domains of interest (telecommunications, embedded systems, System-on-Chip, real time computing, aerospace, automotive, etc.) addressing the inability of third-generation languages to alleviate the complexity of platforms and express domain concepts effectively [35].

MBD emphasizes that software systems should be designed at a high abstraction level and then incrementally refined to contain more specific and detailed information, ending with the implementation of the system in a given platform. MBD principles propose the automation of parts of these refinements through automatic model transformations in order to increase both the productivity and the quality of the developed systems. Model transformations are currently a key research topic in the MBD context. The main research interest lies on vertical transformations, i.e., transformations between models of different levels of abstraction such as PIM (platform independent model) level, PSM (platform specific model) level, and code level of the OMG Model-driven Architecture (MDA) [17]. Another important aspect are horizontal transformations, where models are translated into other models on the same level of abstraction.

In [31], we presented a MBD-based methodology for embedded systems that we defined according to the platform-based design principles $[45,15]$ and by exploiting the OMG MDA as framework for MBD. The de- 
sign methodology is based on the UML2, on a SystemC UML profile for the hardware parts, and on a multithread C UML profile for the software parts. Both the two UML profiles are consistent sets of modeling constructs designed to lift, respectively, constructs (both structural and behavioral) of the SystemC and C coding languages to the UML modeling level. The design methodology, supported by a modeling environment, allows modeling the system at higher levels of abstraction from a functional level to Register Transfer Level (RTL), handling both the hardware architecture with the Hardware-dependent software (HdS) - i.e. all software that is directly dependent on the underlying hardware - and the (hardware-independent) application software. In [33], we presented the development process UPES (Unified Process for Embedded Systems), as extension of the well-known software engineering UP (Unified Process) [2] from the authors of the UML, to foster our design methodology. UPES includes a sub-process, called UPSoC (Unified Process for SoC), which combines all involved notations together in a systematic and seamless manner for the HdS refinement flow.

In this paper, we show how the UPES/UPSoC processes are based on vertical and horizontal model transformations from abstract models toward refined (but still abstract) models and/or software code models. For the UPSoC sub-process, we show how well-established abstraction/refinement patterns coming from hardware or system engineering and till now only used at code level, can be managed as model-to-model transformation steps and therefore applied at UML level, by the use of the SystemC UML profile, along the modeling process from a high functional level model down to a RTL model. We also show how MBD principles are applied to provide the automation of model-to-code transformations from abstract UML models toward detailed SystemC code.

The remainder of this paper is organized as follows. Sections 2 and 3 describe the design process UPES and its sub-process UPSoC, respectively. Sect. 4 gives an overview of the notations adopted in the UPSoC process, while Sect. 5 provides a brief description of the design environment. Sect. 6 presents automatic model transformations used in the UPSoC process. Sect. 7 provides some related work. Sect. 8 concludes the paper and sketches some future directions of our contribution.

\section{The UPES (Unified Process for ES)}

The UPES process drives the designers during the UML modeling activity from the analysis of the informal requirements to a high-level functional model of the system, down to a RTL model, by supporting current in- dustry best practice and the platform-based design principles $[45,15]$. The UPES process is an MDA style of a Y-development cycle (see Fig. 1). It consists on one side of the conventional UP process for software development to define a first executable model of the system (the Application model or System model), and on the other side of the selection process of an available hardware platform (the Platform model) modeled with an appropriate language (e.g., a particular UML extension for SoC design).

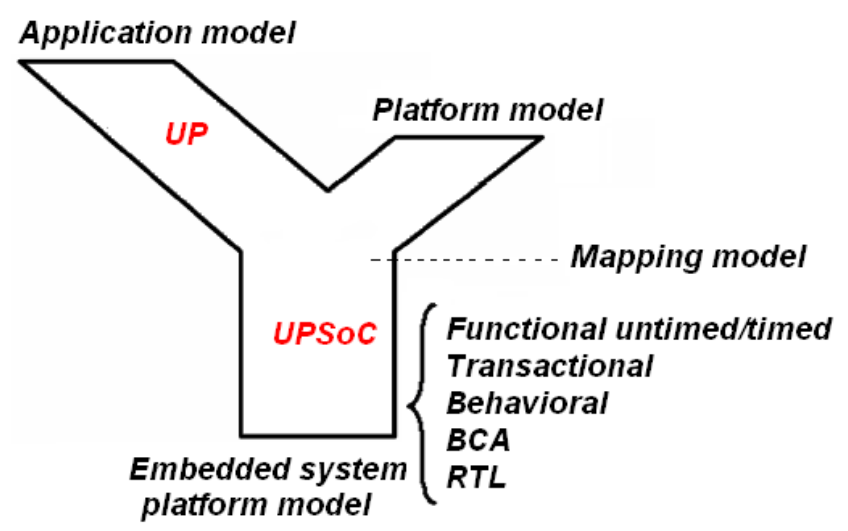

Fig. 1 The UPES Y-chart

The intersection, which is intended as a model weaving operation in the model-based context, is the mapping of the application model on the given platform model to establish semantic links between models at specific join points. As input, this task requires also a reference model of the mapping (the Mapping model or Weaving model) to try, which is specified in terms of UML component and deployment diagrams to denote and annotate the partitioning of the original system in HW and SW components. This mapping model establishes the relationships (join points) of the platform resources and services with the application-level functional components. The UPSoC sub-process, an UP extension for SoC design (see next section), is then followed to accurately refine the embedded system platform from a functional level to the RTL level.

\section{The UPSoC (Unified Process for SoC)}

The UPSoC drives system designers during the refinement of the embedded software, after the mapping phase, and therefore after the system components assigned to the HW partition have been mapped directly onto the $\mathrm{HW}$ resources of the selected platform. The UPSoC can be also adopted in a stand-alone way also by plat- 


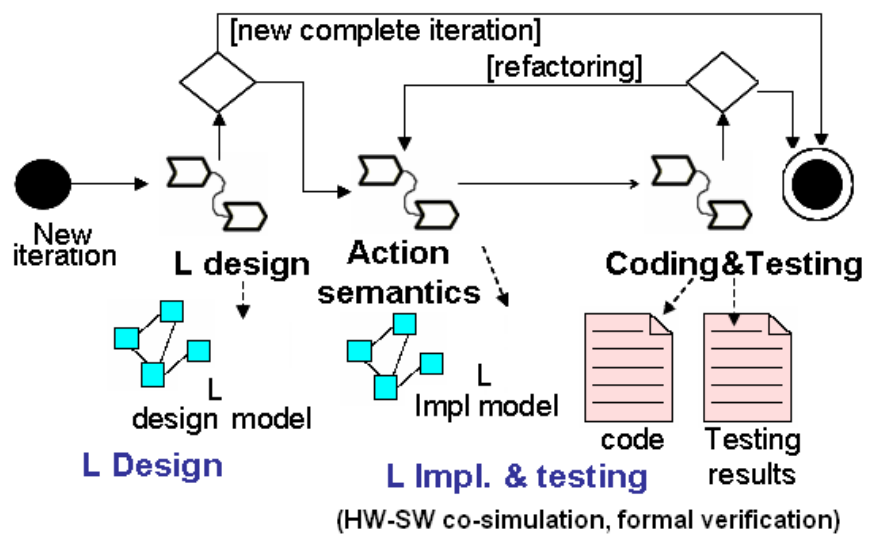

Fig. 2 The UPSoC - L- Design, Implementation and Testing workflow

form providers to deliver off-line models of platforms ${ }^{1}$ by designing from scratch an abstract hardware platform - from the analysis of informal requirements about the architecture elements (HW resources and processing elements) - or to directly design it at a desired level of abstraction. The model of this generic platform could be then reused and targeted accordingly to implement a specific hardware platform where to embed a given system. In both cases, by using e.g. the UML profile for SystemC $[34,30]$ that provides in a graphical way all modelling elements for the design of the hardware components, the hardware platform can be modelled at different abstraction levels: functional untimed/timed, transactional (TLM), behavioral, bus cycle accurate, and RTL (see Fig. 1).

The UPSoC adapts the UP to the SoC domain by extending the design and implementation workflows, while keeping the use case and analysis workflows ${ }^{2}$. Useful considerations about use case analysis in the context of systems engineering and SoC design can be found in [43]. The design and implementation models in the UPSoC can be defined at different abstraction levels and with different modeling guidelines. Fig. 2 shows the workflow schema for these models using the SPEM [38] notation. The letter $L$ in the figure denotes a specific level of abstraction from the set ffunctional, TLM, Behavioral, BCA, RTL\}; therefore, there are five design/implementation workflows. As in the UP, also a Testing model (not shown in the figure) can be pro-

1 Detailed platform models comprise a structural view (provided, e.g., by UML composite structure diagrams) and a behavioral one. Component and deployment diagrams can be used then to provide a black-box view of the API layer and the microarchitecture layer, respectively.

2 Each UP phase (Inception, Elaboration, Construction, and Transition) usually consists of a number of iterations. During each iteration, several activities, called workflows, are performed in parallel. The core workflows of UP are requirements (or use cases), analysis, design, implementation and testing. vided by creating test cases and test procedures housed outside of the modeling tool, but traced back to the models.

From the TLM level to the RTL, precious refinement guidelines $[40,6]$ can be followed to bridge refined elements with more abstract elements like those regarding the introduction of wrappers and adaptors, and to restrict the design for the hardware modeling. A concise description of each of the five workflows follows.

Functional design/implementation.After analysis, the design phase starts by defining the components of the platform as a set of UML subsystems and interfaces. A subsystem is a type of UML component whose functionality is exposed to the outside environment in the form of one or more interfaces representing operational contracts (a message-based interface communication concept). At the end of the design phase, a draft architecture and its functionality is available.

The architectural design is performed in an incremental way, by creating a subsystem that corresponds to each analysis package (output of the analysis workflow) and then refining those subsystems. UML package diagrams can be used to show the subsystems. Then details are added in each subsystem, dividing each subsystem into a specification and a realization part. The former contains design use cases obtained by refining the use cases of the corresponding analysis package. The latter contains design classes and interfaces coming from the analysis classes and from an analysis of the design use cases. UML class diagrams are built to show interfaces, classes and relationships, and UML composite structure diagrams to represent structural hierarchies using parts with ports as basic structural elements, and the notation of provided/required interfaces to denote interface usage/realization dependencies.

The functional design is then performed by identifying operations and dynamic behavior of each class. For 
this purpose, UML state machine diagrams and UML activity diagrams are used, while UML interaction diagrams are useful to get a better understanding of objects interactions. Finally, subsystems are refined to ensure they are as cohesive and loosely coupled as possible, and that they provide the functionality denoted by their specification part.

The UML model developed in the design phase corresponds to the initial functional untimed model of the embedded system. It describes the pure system functionality, and the structural elements do not correspond to blocks in the physical implementation, no time information is provided, and communication is modeled point to point. In order to make it executable and timed - functional timed model-, the implementation phase has to be carried out, where a precise action semantics and time model have to be adopted. An action (or surface) language can be used, supported by a proper execution engine, and the behavioral diagrams can be enriched with actions expressed according to the adopted action and time semantics. Timing diagrams, and annotate interaction diagrams with time-related constraints ${ }^{3}$ may be also included. Typical execution engines for the SoC design are those used for programming languages like $\mathrm{C} / \mathrm{C}++$, System $\mathrm{C}$, etc. UML profiles targeted to these execution engines and model-to-code transformations provide painless and automatic paths toward these environments.

Transactional design/implementation. At TLM level, the architecture is designed and validated in terms of functionality as characterized by high level block input and output events, and inter-block data transfers. Communication details among processing elements are separated from the details of computation. Communication is modeled by channels, while transaction requests take place by calling interface functions of these channel elements. Transactions are protocols exchanging abstract data between blocks, with associated timing information. Elements of a TLM model can correspond or not to blocks in the physical implementation. Channels'interfaces encapsulate low-level details to enable higher simulation speed than pin-based interfaces and also to speed up the modeling task.

The SoC architecture is designed with IP blocks connected via application program interfaces (APIs) to implementation-independent high-level bus models. System IP components and buses may be modified or replaced with greater ease than at RTL, and simulated

\footnotetext{
3 The SysML modeling language [39] and the MARTE UML profile [37], e.g., provide a set of constructs for expressing timerelated aspects.
}

more than 1,000 times faster. Thus designers can quickly optimize the design to achieve what works best.

To make a transactional model executable (implementation model), UML profiles for languages as SpecC and SystemC supporting the transaction level model can be considered. Similar considerations apply to the next levels.

Behavioral design/implementation. A behavioral mo$d e l$ is pin and functionally accurate at its boundaries. It is not cycle accurate, i.e. internal and $\mathrm{I} / \mathrm{O}$ events are not scheduled in a cycle accurate manner. There is a certain freedom left to implement the model in a certain number of clock cycles.

BCA design/implementation.A Bus Cycle Accurate model is pin accurate and functionally accurate. It is also cycle accurate at its boundaries, i.e. in the number of clock cycles it takes to perform its functionality (I/O interactions are scheduled at the proper clock cycle, internal events are not scheduled).

RTL design/implementation. At RTL level the hardware is described in terms of transfers among registers though functional units (adders, multipliers, ALUs, etc.). The RTL model is accurate at the clock cycle level, both at the boundaries and internally (all actions are scheduled at the appropriate clock cycle), and also pin accurate.

The RTL platform model serves as input to the lower level VLSI design flow, where logic simulation and synthesis are performed leading to the final product.

\section{UPSoC notations}

A brief overview is provided here of the two main notations adopted in the UpSoC sub-process: the SystemC coding language as target language of the model-tocode transformations, and the SystemC UML profile as main modeling notation used at different levels of abstractions and, therefore, source/target language of the model-to-model transformations.

\subsection{SystemC overview}

SystemC [21,1] is an IEEE standard controlled by the major companies in the EDA (Electronic Design Automation) industry. It is a system-level design language intended to support the description and validation of complex systems in an environment completely based on the $\mathrm{C}++$ programming language. 


\begin{tabular}{|c|c|}
\hline $\begin{array}{c}\text { Methodology-Specific } \\
\text { Libraries } \\
\text { Master/Slave Library, etc. }\end{array}$ & $\begin{array}{c}\text { Layered Libraries } \\
\text { Verification Library } \\
\text { Static Dataflow, etc. }\end{array}$ \\
\hline \multicolumn{2}{|c|}{ Primitive Channels } \\
Signal, Mutex, Semaphore, FIFO, etc. \\
\hline $\begin{array}{c}\text { Core Language } \\
\text { Modules } \\
\text { Ports } \\
\begin{array}{c}\text { Processes } \\
\text { Interfaces } \\
\text { Channels } \\
\text { Events }\end{array} \\
\text { Data Types } \\
\text { 4-valued Logic type } \\
\text { Bits and Bit Vectors } \\
\text { Arbitrary Precision Integers } \\
\text { Fixed-point types } \\
\text { C++ user-defined types }\end{array}$ \\
Event-driven simulation & C++ Language Standard \\
\hline \hline \multicolumn{2}{|c|}{ C+ }
\end{tabular}

Fig. 3 SystemC Language Architecture

SystemC is defined in terms of a $\mathrm{C}++$ class library, organized according to a layered-architecture shown in in Fig. 3 (taken from [40]). The Core Language and Data Types are the so called core layer (or layer 0) of the standard SystemC, and consists of the eventbased and discrete-timed SystemC simulation kernel, the core design primitives and data types. The Primitive Channels represents, instead, the layer 1 of SystemC; it comes with a predefined set of interfaces, ports and channels for commonly used communication mechanisms such as signals and fifos. Finally, the external libraries layer on top of the layer 1 are not considered as part of the standard SystemC language.

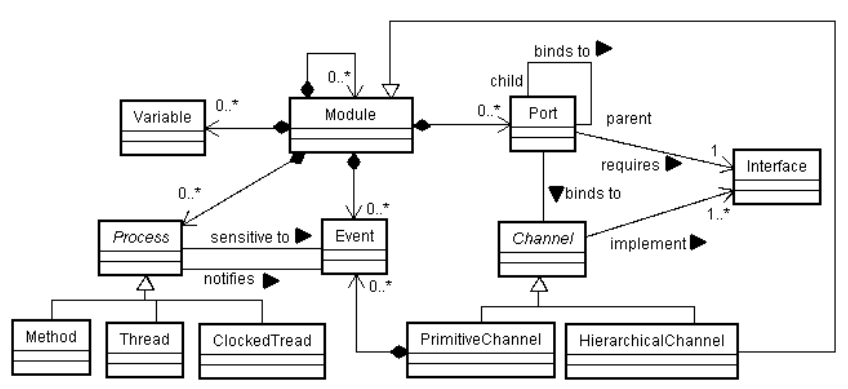

Fig. 4 A simplified SystemC Metamodel

Figure 4 depicts a simplified metamodel (an abstract syntax provided in terms of a class diagram) of the main SystemC terms and concepts. The design of a system in SystemC is essentially given by a containment hierarchy of modules. A module is a container class able to encapsulate structure and functionality of hardware/software blocks. Each module may contain variables as simple data members, ports for communication with the surrounding environment and processes for performing module's functionality and expressing concurrency in the system. Two kinds of processes are available: method processes and thread processes. They run concurrently in the design and may be sensitive to events which are notified by other processes. A port of a module is a proxy object through which the process accesses to a channel interface. The interface defines the set of access functions for a channel, while the channel provides the implementation of these functions to serve as a container to encapsulate the communication of blocks. There are two kinds of channels: primitive channels and hierarchical channels. Primitive channels do not exhibit any visible structure, do not contain processes, and cannot (directly) access other primitive channels. A hierarchical channel is a module, i.e., it can have structure, it can contain processes, and it can directly access other channels.

In 2006, SystemC received a major revision (2.2) and became IEEE Standard [1]. This last revision includes new structural and behavioural features for transaction-level modeling (TLM) according to the OSCI TLM standard.

\subsection{The SystemC UML profile}

The UML2 profile for SystemC [34] is a consistent set of modelling constructs designed to lift both structural and behavioral constructs of the SystemC coding language (including events and time features) to the UML modeling level.

A UML2 profile is a set of stereotypes. Each stereotype defines how the syntax and the semantics of an existing UML2 construct (a class of the UML2 metamodel) is extended for a specific domain terminology or purpose. A stereotype can define additional semantic constraints - the well-formedness rules expressed in the OCL (Object Constraint Language) [20] over the base metaclass - to enforce a semantic restriction of the extended modeling element, as well as tags to state additional properties. Fig. 5 shows an example of stereotype definition for the SystemC sc_port together with an example of OCL constraint. At model level, when a stereotype is applied to a model element (an instance of a UML metaclass), an instance of a stereotype is linked to the model element. From a notational point of view, the name of the stereotype is shown within a pair of guillemets above or before the name of the model element and the eventual tagged values displayed inside or close as name-value pairs. Examples of stereotypes application are provided here (see Fig. 6, e.g., for the sc_port stereotype) and in Sect. 6.1.

The SystemC UML profile is defined at two distinct levels - the SystemC core layer (or layer 0) and the SystemC layer of predefined channels, ports and interfaces (or layer 1) - reflecting the layered-architecture of SystemC. 


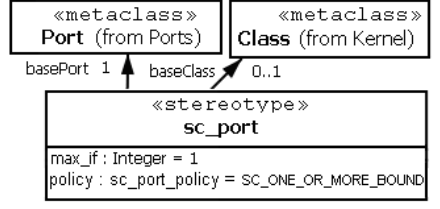

English: A port can have exactly one required interface and no provided interfaces. $\boldsymbol{O C L}$ : basePort.required->size( $)=1$ and basePort.provided->size ()$=0$

Fig. 5 sc_port stereotype

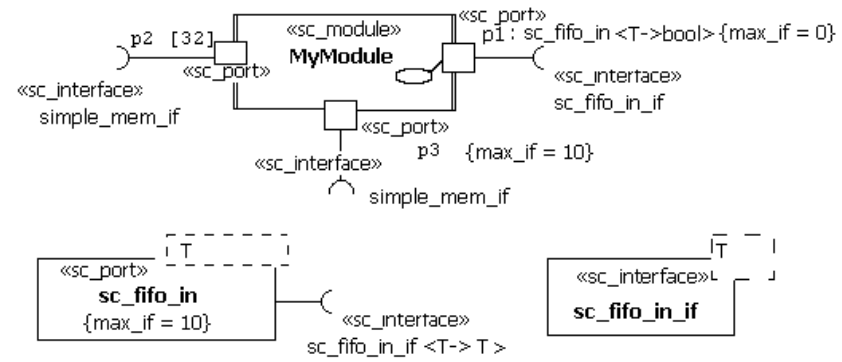

Fig. 6 Modules, ports and interfaces

The core layer - the basic SystemC profile - is the foundation upon which specific libraries of model elements or also other modeling constructs can be defined. It is logically structured as follows.

A Structure and Communication part defines stereotypes for the SystemC building constructs (modules, interfaces, ports and channels) for use in UML structural diagrams like UML class diagrams and composite structure diagrams. UML class diagrams are used to define modules, interfaces and channels. Fig. 6 shows an example of a SystemC module exposing a multiport, an array port, and a simple port, together with the port type and the interface definitions of the simple port. The internal structure of composite modules, especially the one of the topmost level module (representing the structure of the overall system), is captured by UML composite structure diagrams. From these diagrams several UML object diagrams can be created to describe different configuration scenarios. This separation allows the (also partial) specification of different $\mathrm{HW}$ platforms as instances of the same parametric model (i.e. the composite structure diagram). Concrete examples of composite modules are provided in Sect. 6.1 .

A Behavior and Synchronization part defines special state and action stereotypes which lead to an extension of the UML state machines, the SystemC Process State Machines [29]. This formalism has been appositely included in the profile definition to model the control flow and the reactive behavior of SystemC processes (methods and threads) within modules. A finite number of abstract behavior patterns of state machines

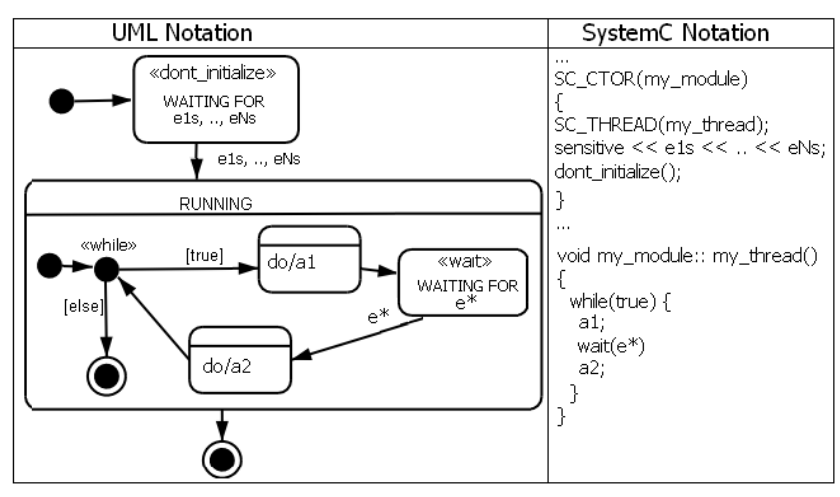

Fig. 7 A thread process pattern

\begin{tabular}{|c|c|}
\hline SystemC & UML Notation \\
\hline $\begin{array}{c}\text { wait }(e) \\
\text { wait for an event } e\end{array}$ & $\begin{array}{c}\text { "wait» } \\
\text { waiting for e }\end{array}$ \\
\hline $\begin{array}{l}\text { wait }(t) \\
\text { wait for } t \text { time units }\end{array}$ & $\begin{array}{c}\text { "wait» } \\
\text { waiting for } t\end{array}$ \\
\hline $\begin{array}{l}\text { wait }(e, t) \\
\text { wait for an event with timeout }\end{array}$ & $\begin{array}{c}\text { "wait» } \\
\text { waiting for e with } \\
\text { timeout } t \\
\end{array}$ \\
\hline $\begin{array}{l}\text { wait }(e 1 \& \ldots \& \in N) \\
\text { wait for an AND-list of events }\end{array}$ & $\begin{array}{c}\mathbf{e}_{1}, \ldots, \mathbf{e}_{N} \\
\text { «and }\end{array}$ waiting for $e_{1} \& \ldots \& e_{N}$ \\
\hline $\begin{array}{c}\text { wait }(e 1|\ldots| e N) \\
\text { wait for an OR-list of events }\end{array}$ & $\begin{array}{c}\text { "wait } t \\
\text { waiting for } e_{1}|\ldots| e_{N}\end{array}$ \\
\hline $\begin{array}{l}\text { wait }(t, e 1 \& \ldots \& \in N) \\
\text { wait for an AND-list of events } \\
\text { with timeout }\end{array}$ & - \\
\hline $\begin{array}{c}\text { wait }(t, e 1|\ldots| e N) \\
\text { wait for an OR-list of events } \\
\text { with timeout }\end{array}$ & $\begin{array}{c}\text { "wait» } \\
\text { waiting for } e_{1}|\ldots| e_{N}\end{array}$ \\
\hline $\begin{array}{l}\text { wait () } \\
\text { wait for static sensitivity }\end{array}$ & "static_wait $t$ " \\
\hline
\end{tabular}

Fig. 8 Dynamic Sensitivity of a Thread

have been identified. Fig. 7 depicts one of these behavior patterns together with the corresponding SystemC pseudo-code for a thread that: (i) is not initialized, (ii) has both a static (the event list $e_{1 s}, \ldots, e_{N s}$ ) and a dynamic sensitivity (the wait state), and (iii) runs continuously (by the infinite while loop). Note that the notation used for the wait state in the pattern in Fig. 7 stands for a shortcut to represent a generic wait (e*) call where the event e* matches one of the cases reported in Fig. 8. Moreover, activities a1 and a2 stand for blocks of sequential (or not) code without wait statements.

It should be noted that the state machine pattern depicted in Fig. 7 can be more complex in case of one or more wait statements are enclosed in the scope of one or more nested control structures. In this case, as part of the UML profile for SystemC, the control structures while, if, etc. need to be explicitly represented 


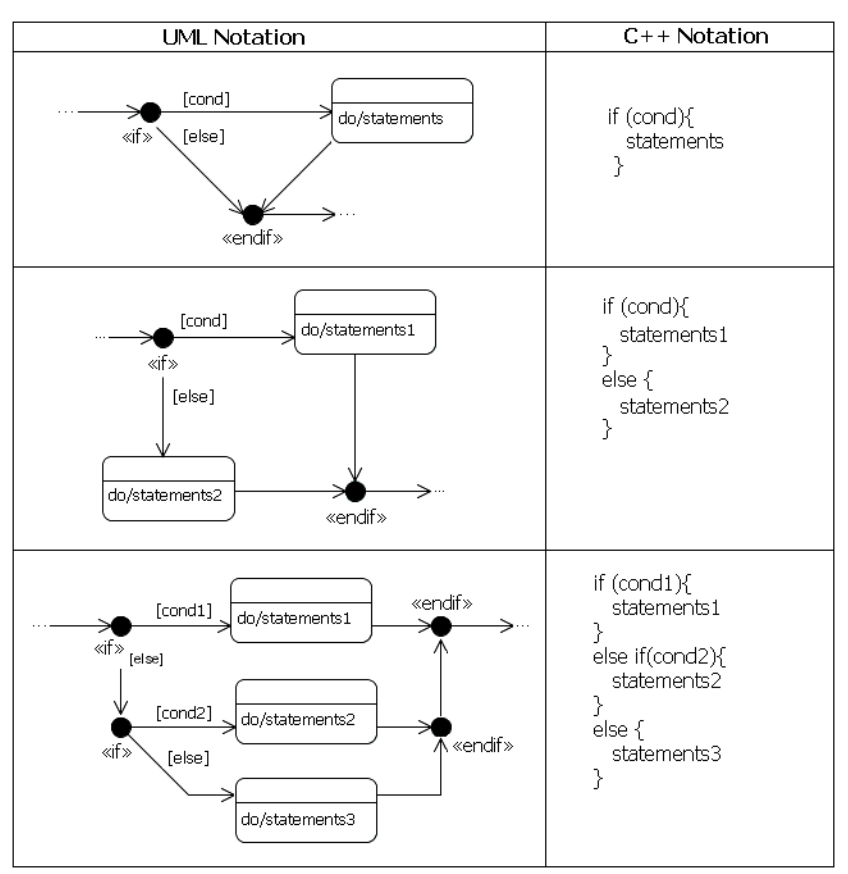

Fig. 9 Conditional controls

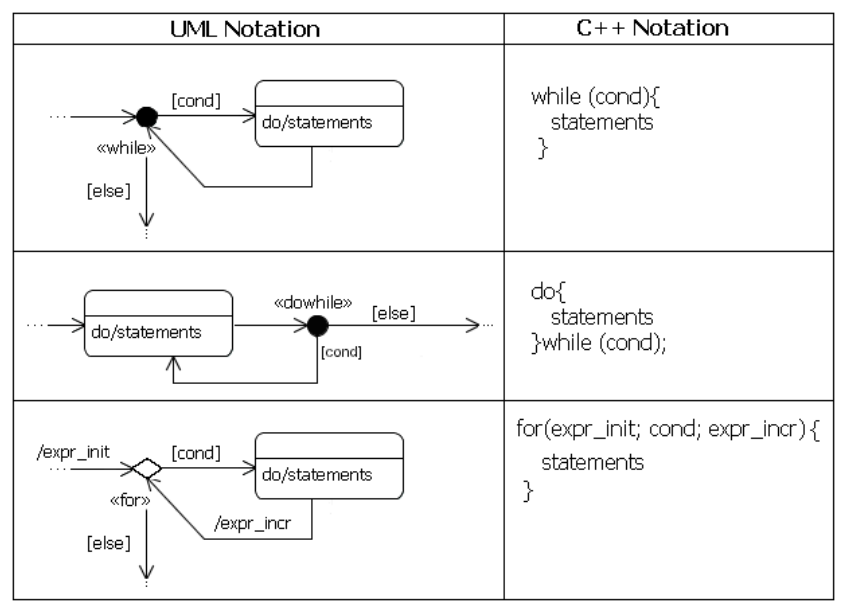

Fig. 10 Loop controls

in terms of special stereotyped junction or choice pseudostates ${ }^{4}$ combined together in order to stand out the state-like representation of the wait calls. The conditional control and loop controls, e.g., can be modeled as shown in Fig. 9 and in Fig. Fig. 10, respectively. These stereotypes allow us to effectively generate code from state diagrams in a style that reflects the nature of constructs of the target implementation language, despite to well know techniques (like state pattern, stable table pattern, etc.) currently used to achieve this goal.

\footnotetext{
4 Choice pseudostates must be used in place of junction pseudostates whenever the head condition of the while loop is a function of the results of prior actions performed in the same run-tocompletion step.
}

A Data types part defines a UML class library to represent the set of SystemC data types.

In addition, predefined channels, ports and interfaces of the layer 1 of SystemC are provided either as a UML class library, modeled with the basic stereotypes of the SystemC core layer, or as a group of stand alone stereotypes - the extended System C profile - specializing the basic profile.

An extended version of the SystemC UML profile encapsulating some new structural and behavioural features (i.e., dynamic processes and fork/join synchronization mechanisms) necessary to model a TLM library conforming to the OSCI SystemC TLM library, was presented in [3].

\section{UPSoC design tool}

For industrial application, the availability of appropriate tool support is crucial. In order to cover the full spectrum of the UPSoC by using $\mathrm{C} / \mathrm{C}++$ and SystemC as action languages, we developed a design tool working as front-end for consolidated lower level co-design tools.

Fig. 11 shows the tool architecture. Components visualized inside dashed lines are still under development. The tool consists of two major parts: a development kit (DK) with design and development components, and a runtime environment ( $\mathrm{RE}$ ) represented by the SystemC execution engine.

The DK consists of a UML2 modeler supporting the SystemC UML profile, translators for the forward/reverse engineering to/from $\mathrm{C} / \mathrm{C}++/$ System $\mathrm{C}$, and an abstraction/refinement evaluator to guarantee traceability and correctness along the refinement process from the high-level abstract description to the final implementation. A more detailed description of the tool is provided in [32].

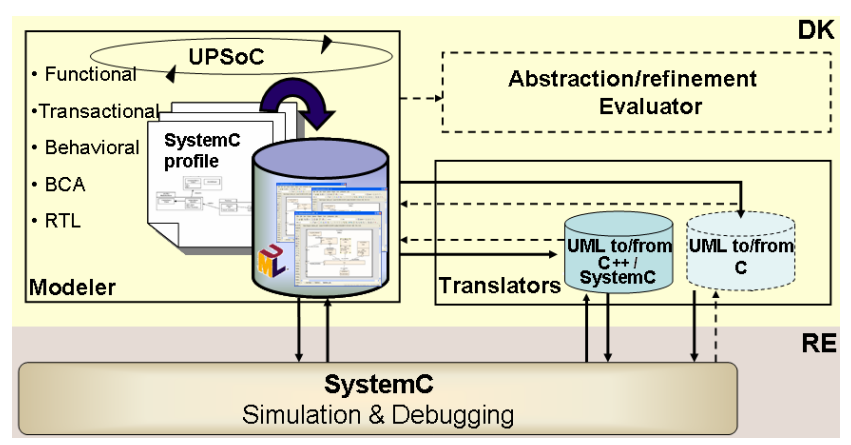

Fig. 11 Tool architecture 


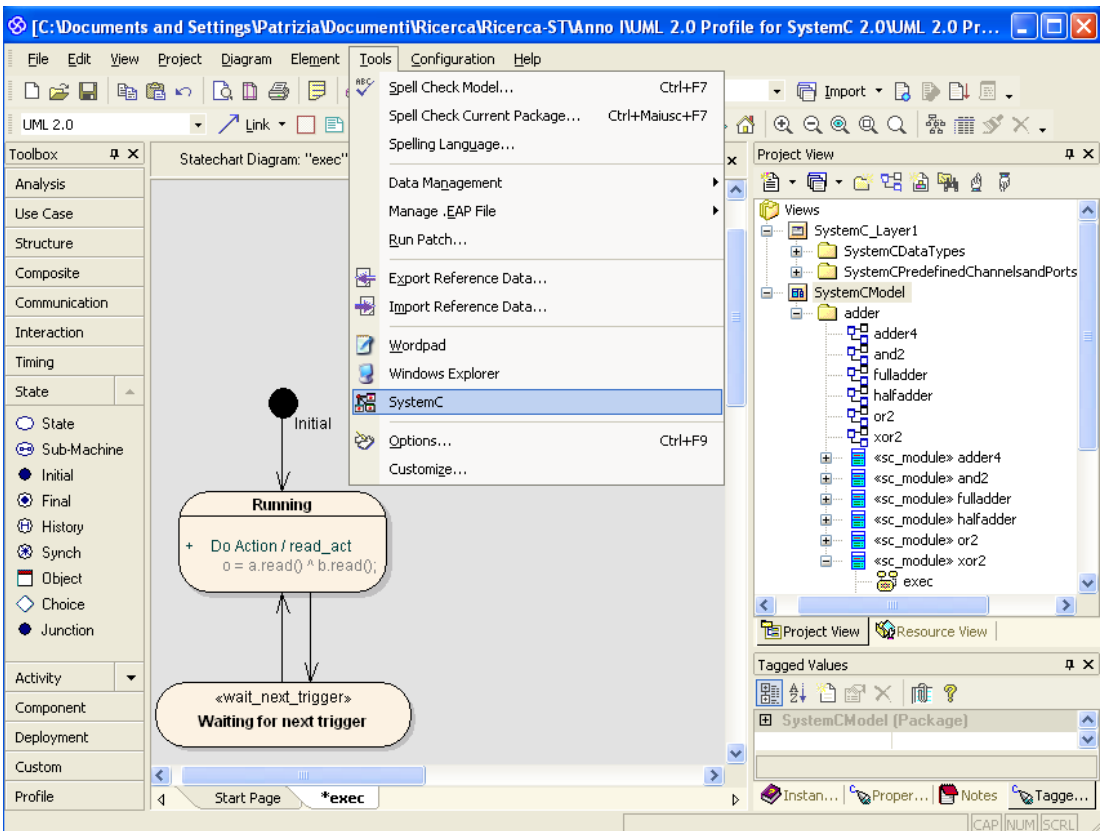

Fig. 12 Generate SystemC code from EA

Initially, the UML2 modeler was based on the commercial Enterprise Architect (EA) tool [10] by SparxSystems. Fig. 12 shows a screenshot of EA. The SystemC data types and predefined channels, interfaces, and ports are modelled with the core stereotypes, and are available in the Project View with the name SystemC_Layer1.

Recently, we have implemented the UML2 modeler also in the Papyrus framework [23]. This last is an open source Eclipse-based UML modeler which supports the UML2 standard as defined by the OMG and allows the integration of model transformation services. These last are handled by transformation engines, like the ATL engine [13] (the one we adopted), developed within the Eclipse Modeling Project [12] as implementation of the OMG QVT [27] standard.

\section{UPSoC model transformations}

The MDA-style separation of models of UPES/UPSoC demands automatic model transformations as support to evolution activities [18] in general, like refinemen$\mathrm{t} /$ abstraction, model refactoring, model inconsistency management, etc.

According to the classification given in [8], for refinement/abstraction purposes we identify two main kinds of model transformations to be adopted specifically in the UPSoC process: model-to-model transformations and model-to-code transformations. They are better explained in the following sections by providing concrete examples.

\subsection{Model-to-model transformations}

Model-to-model transformations of UPSoC allow model refinement along the (not necessarily successive) abstraction levels: functional, transactional, behavioral, $\mathrm{BCA}$, and RTL. These transformations must be intended as vertical transformations as they imply a change of the level of abstraction. Each model is here intended as an instance of the SystemC UML profile metamodel, and therefore is an implementation model with a fixed action semantics.

In the model-to-model category, we adopt an hybrid approach based on both direct-manipulation and structure-driven approach [8]. The idea is to collect and reuse precise abstraction/refinement transformation patterns, coming from industry best practices. The transformation patterns are once for all proved correct and complete, and can be used for guiding the refinement process or for pointing out missing elements in a refinement.

We have implemented some of these patterns in the EA-based environment by using the EA MDA transformers, and in the Papyrus-based environment using the ATL engine. By applying the model transformations defined for a given pattern, a UML system design may automatically and correctly evolve to an abstract/refined model reflecting the abstraction/refinement rules defined for the applied pattern. Below, we provide an example of communication refinement from functional level to RTL level based on model transformations. 


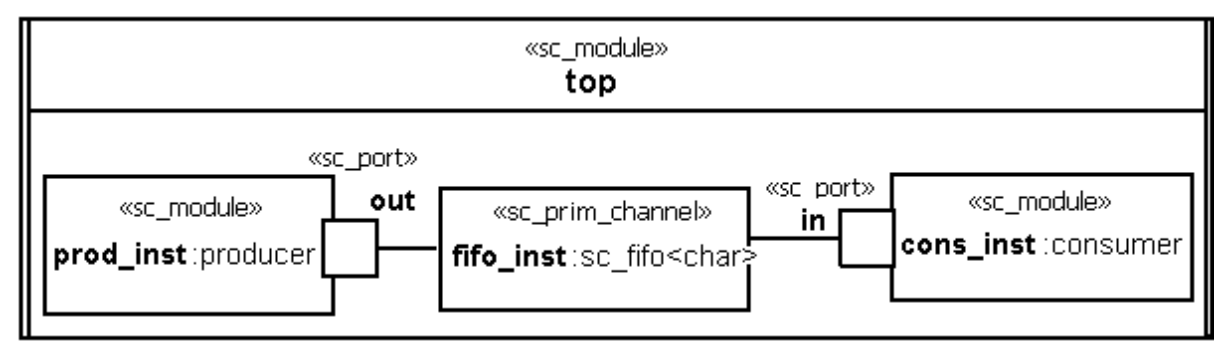

Fig. 13 A producer/consumer design

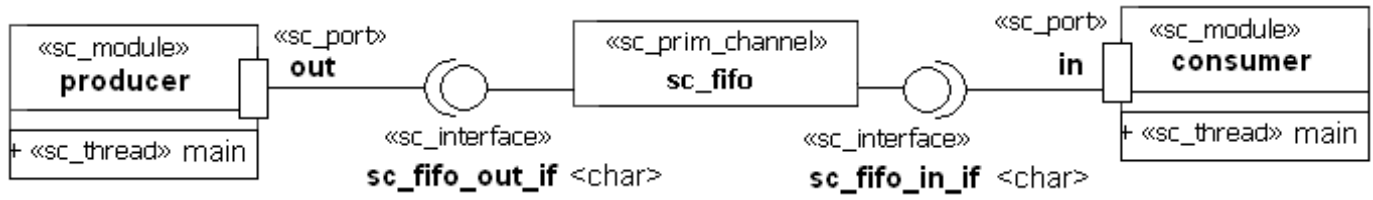

Fig. 14 Producer/consumer modules communicating via a primitive FIFO

\subsubsection{Communication refinement}

In general, in the refinement process we do not only refine the model's internal structure, its timing, or the data-types being used; we also need to think about how components communicate with their environment. Communication refinement refers to mapping an abstract communication protocol into an actual implementation related to a given target architecture. To give an idea on how to perform refinement at UML level by model-to-model transformations, we show here how to apply communication refinement patterns to a functional timed model leading to a RTL model.

We clarify the process of communication refinement in a general way. To this purpose, we assume to have two high level modules M1 and M2 communicating over a channel $C$ via some abstract protocol. As suggested in [40], one possible approach to refining this basic communication scenario toward an implementation consists of the following steps:

1. Select an appropriate communication scheme to implement;

2. Replace the abstract communication channel $\mathrm{C}$ with a refined one $C_{\text {Refined }}$ realizing the selected communication protocol;

3. Enable the communication of the modules $\mathrm{M} 1$ and $\mathrm{M} 2$ over $\mathrm{C}_{\text {Refined }}$ by either:

(a) wrapping $C_{\text {Refined }}$ in a way that the resulting channel $C_{W \text { rapped }}$ provides the interfaces required by $\mathrm{M} 1$ and $\mathrm{M} 2$ (wrapping), or

(b) refining $\mathrm{M} 1$ into $\mathrm{M} 1_{\text {Refined }}$ and $\mathrm{M} 2$ into $\mathrm{M} 2$ Refined in order their required interfaces match the ones provided by $\mathrm{C}_{\text {Refined }}$ (adapter-merging).

The two cases (not the only ones) are similar. Both include an intermediate step to build two further modules - i.e. two hierarchical channels called adapters - to map one interface to another: one, let us say $\mathrm{A} 1$, between $\mathrm{M} 1$ and $\mathrm{C}_{\text {Refined }}$, and one, let us say $\mathrm{A} 2$, between $\mathrm{C}_{\text {Refined }}$ and $\mathrm{M} 2$. In the case (a), the resulting channel $C_{\text {Wrapped }}$ encloses $C_{\text {Refined }}$ and the two adapters; while, in the case (b) these adapters are merged to the calling modules $\mathrm{M} 1$ and $\mathrm{M} 2$ resulting in the refined modules $\mathrm{M} 1_{\text {Refined }}$ and $\mathrm{M} 2_{\text {Refined. }}$. Deciding whether to use wrapping or merging depends on the methodology and chosen target architecture.

Example We show here how to apply the communication refinement strategy described above to a simple abstract (functional timed) producer/consumer system taken from [40]. The design of a producer/consumer module that writes and reads characters to/from a FIFO channel is shown by the UML composite structure diagram in Fig. 13. The top composite module is defined to contain one instance of the consumer module, one instance of the producer module, and one FIFO channel instance. The FIFO channel permits to store characters by means of blocking read and write interfaces, such that characters are always reliably delivered. Two processes, the producer and the consumer (see the thread processes main within the producer and the consumer modules in Fig. 14), respectively feed and read the FIFO. The producer module writes data through its out port into the FIFO by a sc fifo_out if interface, the consumer module reads data from the FIFO through its in port by the sc_fifo_in_if interface. These two interfaces are implemented by the FIFO channel (see the sc_fifo channel in Fig. 14). Because of the blocking nature of the sc_fifo read/write operations, all data are reliably delivered despite the varying rates of production and consumption.

Now, let's assume to replace the (abstract) FIFO instance above with a (refined) model of a clocked RTL 


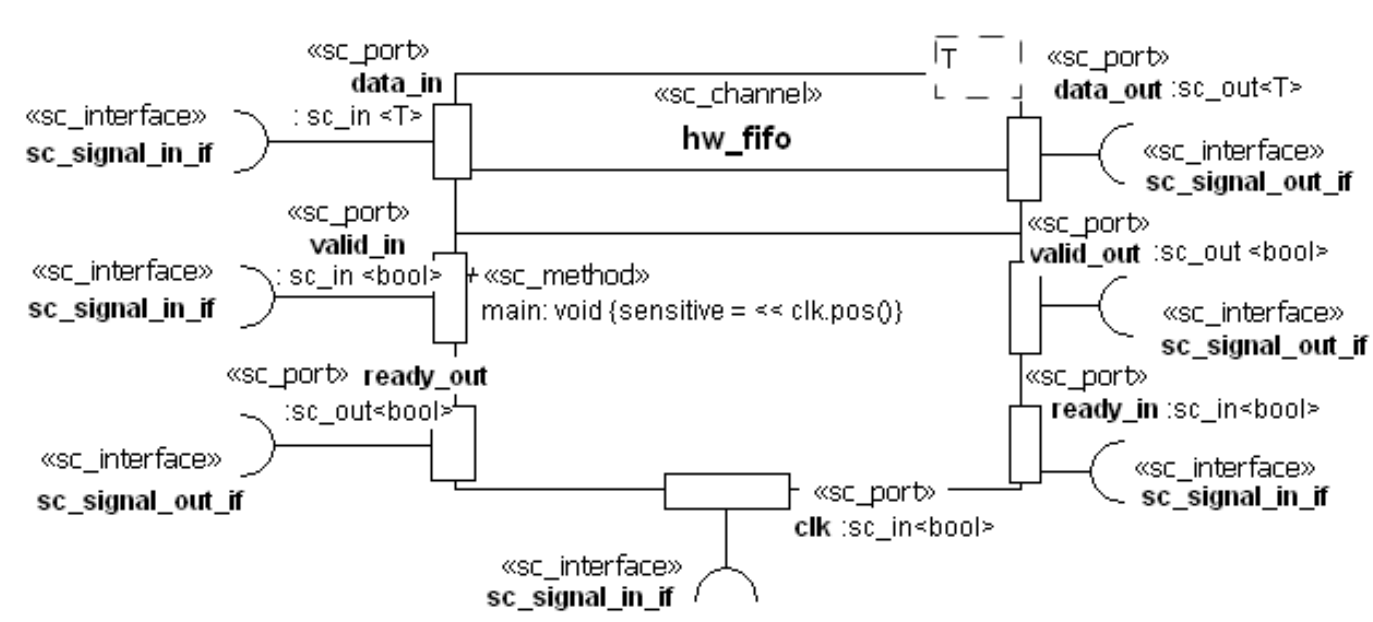

Fig. 15 Clocked RTL HW FIFO

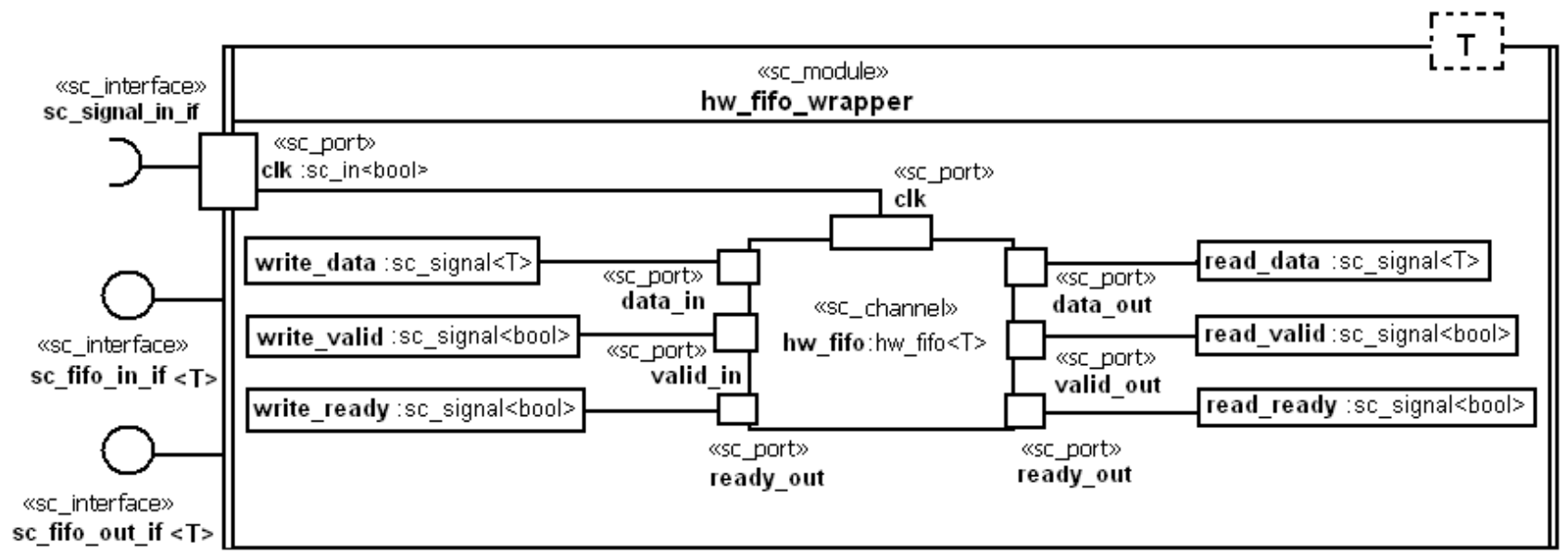

Fig. 16 The hw_fifo_wrapper hierarchical channel

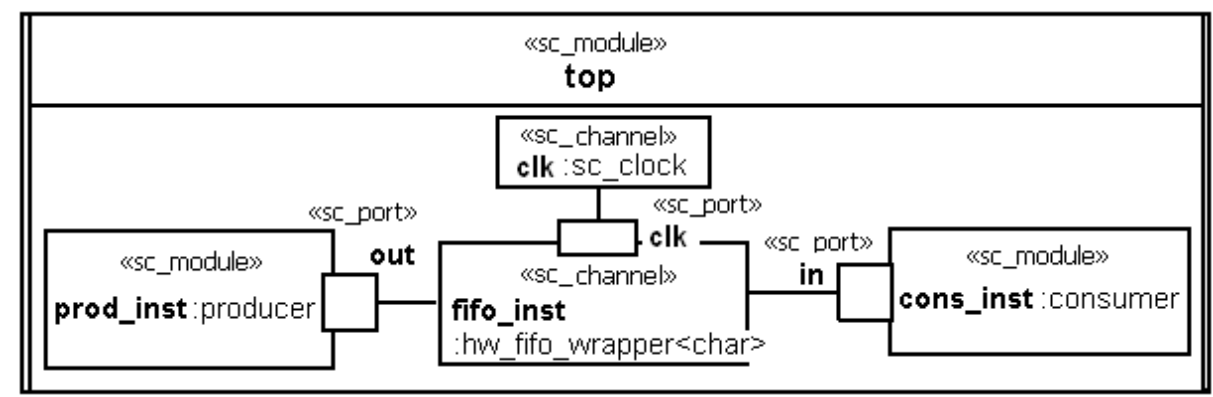

Fig. 17 A clocked producer/consumer design

hardware FIFO named hw_fifo $\langle\mathrm{T}\rangle$ for an hardware implementation. The new hardware FIFO uses a signallevel ready/valid handshake protocol for both the FIFO input and output (see Fig. 15). It should be noted that we cannot use hw fifo directly in place of sc fifo, since the former does not provide any interfaces at all, but has ports that connect to signals, i.e. has ports that use the sc_signal_in_if and sc_signal_out_if interfaces.

Following the wrapper-based approach 3.b described above, we can define a hierarchical channel hw fifo - wrapper $<\mathrm{T}>\left(\mathrm{C}_{\text {Wrapped }}\right)$ implementing the interfaces sc_fifo_out_if and sc_fifo_in_if and containing an instance of $\mathrm{hw}_{-}$fifo $<\mathrm{T}>\left(\mathrm{C}_{\text {Refined }}\right)$. In addition, it contains SC_signal instances to interface with hw_fifo $\langle\mathrm{T}\rangle$ and a clock port (since hw_fifo $\langle\mathrm{T}>$ has also a clock port) to feed in the clock signal to the hw_fifo $\langle\mathrm{T}\rangle$ instance (see Fig. 16). Finally, we need to add a hardware clock instance in the top-level design to drive the additional clock port that is now on the hw_fifo_wrapper $\langle\mathrm{T}\rangle$ instance (see Fig. 17). The hw_fifo_wrap- 
per $\langle\mathrm{T}\rangle$ implements the required signal-level ready/valid handshake protocol whenever a read or write operation occurs; this protocol will properly suspend read or write transactions if $\mathrm{hw}$ fifo $\langle\mathrm{T}\rangle$ is not ready to complete the operation. Details on the SystemC code can be found in [40].

\subsection{Model-to-code transformations}

Model-to-code transformations are primarily aimed at providing executable models at a fixed abstraction level. They must be intended as horizontal transformations creating program text in the SystemC implementation language.

In the model-to-code category, we distinguish between visitor-based and template-based approaches. Visitor-based simply means that the model is navigated and code is generated for each element visited according to specific rules describing for each meta-concept what code to generate and which elements to visit next. Template-based approaches use target text that includes place holders that are to be replaced with concrete data in concrete template instances. Rules in such templatebased approaches usually access information in the source model using the left hand side of a rule and give a template as right hand side that incorporates this data in a piece of target code.

We adopted a visitor-based approach for the EAbased environment. The EA supports forward/reverse engineering to/from $\mathrm{C}++$. We developed an EA addin (based on the EA automation and scripting interface and on the Windows OLE Automation ActiveX technology) which exploits the added semantics in the SystemC UML profile to generate complete SystemC code (full code generation) from input models (for both the structural and behavioral views) developed with the EA-based UML modeler.

The EA-based code generator traverses all class diagrams and for every encountered class it produces a $\mathrm{C}++$ header file (.h); this happens for both the definition of SystemC classes (modules, channels and interfaces) and simple $\mathrm{C}++$ classes, allowing a mixed design style. Classes contain fields and methods. For each method it is possible to describe its behavior either as an inline code description or as a SystemC process state machine diagram. Each process state machine, therefore, contributes to the generation and enrichment of a body file (.cpp) containing the implementation code of all methods of a class or module or channel.

Moreover, by an analysis of the composite structure diagram associated to a module to describe its internal structure (especially the one of the topmost level module, which represents the structure of the overall system), it is possible to determine how internal parts are connected to each other in the module constructors in the header file. Finally, object diagrams may be seen as instances of the composite structure diagrams, useful to model particular initializations of the system components for the construction of appropriate test benches.

The header file producer.h, e.g., for the producer module (see Fig. 14) would contain the declaration of the producer class together with the declaration of the main thread process, as reported in Listing 1.

The body section (file producer.cpp in Fig. 18) is determined by the thread state machine main (see Fig. 18), where the $\mathrm{C}++$ notation is put on top of the UML action semantics and the control structures are directly derived from the stereotyped pseudostates while, if-then, etc., of the state machine diagram.

We have been applying similar code engineering techniques to the Papyrus-based environment, but following a template-based approach by relying on JET and Acceleo technologies. Compared to visitor-based approaches, template-based approaches have the advantage of reusing pieces of code and thus template-based tools are less error prone than visitor-based ones.

Listing 1 producer.h

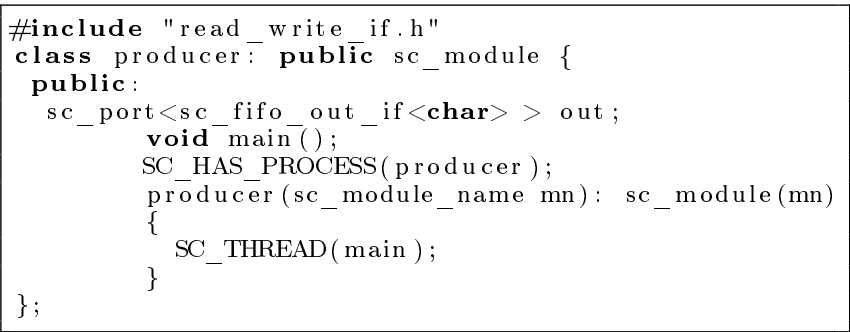

\section{Related work}

In this paper, we provide guidance on how to integrate a UP style of software development into the overall process for Embedded Systems. The UPES reflects current industry best practices and follows the platform-based design principles $[45,15]$. For the UPES definition, we have been taking in consideration the work experiences in $[15,11,46,44]$ as first contributions to the definition of a development process for the Embedded Systems domain based on abstract and executable models and on the System-on-Chip (SoC) paradigm for the softwarehardware convergence.

The possibility to use UML 1.x for system design [16] started since 1999, but the general opinion at that time was that UML was not mature enough as a system design language. Nevertheless significant industrial experiences using UML in a system design process soon 

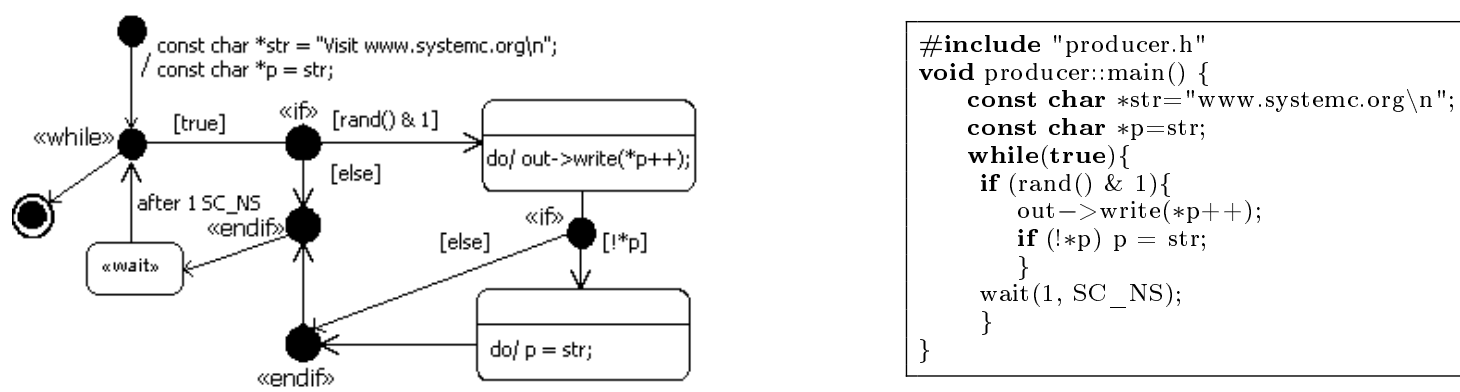

Fig. 18 Producer's main thread state machine and its SystemC code

started leading to the first results in design methodology, such as the one in [41] that was applied to an internal project for the development of a OFDM Wireless LAN chipset. In this project SystemC was used to provide executable models.

More integrated design methodologies were later developed. The authors of [28] propose a methodology using the UML for the specification and validation of SoC design. They define a flow, parallel to the implementation flow, which is focused on high-level specs capture and validation. In [15], a UML profile for a platform-based approach to embedded software development is presented. It includes stereotypes to represent platform services and resources that can be assembled together. The authors also present a design methodology supported by a design environment, called Metropolis, where a set of UML diagrams (use cases, classes, state machines, activity and sequence diagrams) can be used to capture the functionality and then refine it by adding models of computation.

Another approach to the unification of UML and SoC design is the HASoC (Hardware and Software Objects on Chip) [11] methodology. It is based on the UML-RT profile [36] and on the RUP process [14]. The design process starts with an uncommitted model and after a committed model is derived by partitioning the system into software and hardware, and then mapped onto a system platform. From these models a SystemC skeleton code can be also generated, but to provide a finer degree of behavioral validation, detailed $\mathrm{C}++$ code must be added by hand to the skeleton code. All the works mentioned above could greatly benefit from the use of UML2.

In [9], the authors present a model-driven framework called ModES (Model-driven Design of Embedded Systems) made of metamodels definition and APIs to integrate, by model transformations, several modelbased design tools. However, this framework is more related to the design space exploration at a high abstraction level than to model refinement, model validation, and automatic code generation from models, which are, instead, our main concerns.

SysML [39] is a conservative extension of UML 2.0 for a domain- neutral representation (i.e. a PIM model as in MDA [17]) of system engineering applications. It can be involved at the beginning of the design process, in place of the UML, for the requirements, analysis, and functional design workflows. So it is in agreement with our UML profile for SystemC, which can be thought (and effectively made) as customization of SysML rather than UML. Unluckily, when we started the SysML specification was not yet finalized and there were no tools yet supporting it. Similar considerations apply also to the recent MARTE (Modeling and Analysis of Real-Time Embedded Systems) profile initiative [37].

The standardization proposal [42] by Fujitsu, in collaboration with IBM and NEC, has evident similarities with our SystemC UML profile, like the choice of SystemC as a target implementation language. However, their profile does not provide building blocks for behavior modeling and any time model.

Some other proposals already exist about extensions of UML towards $\mathrm{C} / \mathrm{C}++/$ SystemC. All have in common the use of UML stereotypes for SystemC constructs, but not rely on a UML profile definition. In this sense, it is appreciable the work in [5] attempting to define a UML profile for SystemC; but, as all the other proposals, it is based on the previous version of UML, UML 1.4. Moreover, in all the proposals we have seen, no code generation, except in [19], from behavioral diagrams is considered.

Refinement, or in general model-to-model transformation, is another key concept in MBD. However, compared with the refinement techniques available for formal methods like Z, B, and ASMs [4], few work has been carried out for modeling languages like UML. Some proposals that we are considering in our process can be found in $[7,25,26,24,22]$. 


\section{Conclusions and future directions}

This paper describes aspects of reusable model-to-model and model-to-code transformations in the context of the UPES/UPSoc processes for embedded system development. This aspect is vital for integrating, synchronizing or transforming models for our design methodology based on the UPES/UPSoC processes.

Today, model transformations are mainly written from scratch. This is in many cases a very time consuming and difficult task. According to the MBD vision, there must be large libraries of reusable model transformations available.

In the future, we aim at identifying characteristics of reusable transformations and ways of achieving reuse by collecting in a library precise abstraction/refinement transformation patterns according to the levels of abstraction: functional, transactional, behavioral, BCA, and RTL. In particular, we are focusing on the TLM to model the communication aspects at a certain number of TLM sub-levels according to the OSCI TLM 1.0 library [21]. We believe the use of fine-grained transformations that are being composed (chaining) would be beneficial, both increasing the productivity and the quality of the developed systems.

\section{References}

1. SystemC Language Reference Manual. IEEE Std 1666-2005, 31 March 2006.

2. J. Arlow and I. Neustadt. UML and the Unified Process. Addison Wesley, 2002

3. S. Bocchio, E. Riccobene, A. Rosti, and P. Scandurra. An Enhanced SystemC UML Profile for Modeling at TransactionLevel. Embedded Systems Specification and Design Languages. E. Villar (ed.), 2008.

4. E. Börger and R. Stärk. Abstract State Machines: A Method for High-Level System Design and Analysis. Springer Verlag, 2003.

5. F. Bruschi and D. Sciuto. SystemC based Design Flow starting from UML Model. In Proc. of European SystemC Users Group Meeting, 2002.

6. L. Cai and D. Gajski. Transaction Level Modeling: an Overview. In Proc. of CODES+ISSS, Newport Beach, California, USA, 2003.

7. The Catalysis process: www.catalys is.org, 1998.

8. Krzysztof Czarnecki and Simon Helsen. Classification of Model Transformation Approaches. In Proc. of 2nd OOPSLA Workshop on Generative Techniques in the Context of Model-Driven Architecture, 2003.

9. Francisco Assis M. do Nascimento, Márcio F. S. Oliveira, and Flávio Rech Wagner. ModES: Embedded Systems Design Methodology and Tools based on MDE. In Fourth International workshop on Model-based Methodologies for Pervasive and Embedded Software (MOMPES'O7). IEEE Press, 2007.

10. The Enterprise Architect tool: www.sparxsystems.com.au/, 2008.

11. M. Edwards and P. Green. UML for hardware and software object modeling. UML for real design of embedded real-time systems, pages 127-147, 2003.
12. Eclipse Modeling Framework. http://www.eclipse.org/ emf /, 2008.

13. Frédéric Jouault, Freddy Allilaire, Jean Bézivin, Ivan Kurtev, and Patrick Valduriez. ATL: a QVT-like transformation language. In OOPSLA 'O6: Companion to the 21st ACM SIGPLAN conference on Object-oriented programming systems, languages, and applications, pages 719-720. ACM, 2006.

14. P. Kruchten. The Rational Unified Process. Addison Wesley, 1999.

15. L. Lavagno, G. Martin, A. Sangiovanni Vincentelli, J. Rabaey, R. Chen, and M. Sgroi. UML and Platform based Design. UML for Real Design of Embedded Real-Time Systems, 2003.

16. G. Martin. UML and VCC. White paper, Cadence Design Systems, Inc, Dec. 1999.

17. OMG. The Model Driven Architecture (MDA). MDA Guide V1.0.1, http://www.omg.org/mda/, 2003.

18. T. Mens, M. Wermelinger, S. Ducasse, S. Demeyer, R. Hirschfeld, and M. Jazayeri. Challenges in Software Evolution. In Proc. of the International Workshop on Software Evolution. IEEE, 2005.

19. K.D. Nguyen, Z. Sun, P.S. Thiagarajan, and W.F. Wong. Model-Driven SoC Design: The UML-SystemC Bridge. UML for SOC Design, 2005.

20. OMG. UML 2.0 OCL Specification, ptc/03-10-14.

21. The Open SystemC Initiative. http://www.systemc.org, 2008.

22. Richard F. Paige, Dimitrios S. Kolovos, and Fiona A.C. Polack. Refinement via Consistency Checking in MDA. In Proc. Refinement Workshop, ENTCS, Surrey, UK, April 2005.

23. Papyrus UML web site. http://www papyrusuml.org, 2008.

24. C. Pons and R-D Kutsche. Using UML-B and U2B for formal refinement of digital components. In Proc. of Forum on specification and design languages, Frankfurt, 2003.

25. C. Pons and R-D Kutsche. Traceability Across Refinement Steps in UML Modeling. In Proc. of the WiSME@UML workshop, 2004.

26. Claudia Pons and Diego Garcia. An OCL-Based Technique for Specifying and Verifying Refinement-Oriented Transformations in MDE. In MoDELS, pages 646-660, 2006.

27. OMG, MOF Query/Views/Transformations, ptc/07-07-07, 2007.

28. Q.Zhu, R.Oishi, T.Hasegawa, T.Nakata. System-on-Chip Validation using UML and CWL. In Proc. of CODES, 2004.

29. E. Riccobene and P. Scandurra. Modelling SystemC Process Behaviour by the UML Method State Machines. In Proc. of RISE'04. Springer, 2004.

30. E. Riccobene, P. Scandurra, A. Rosti, and S. Bocchio. A UML 2.0 profile for SystemC: toward high-level SoC design. In EMSOFT '05: Proceedings of the 5th ACM international conference on Embedded software, pages 138-141. ACM Press, 2005.

31. E. Riccobene, P. Scandurra, A. Rosti, and S. Bocchio. A Model-driven Co-design Flow for Embedded Systems. In FDL '06: Proceedings of Forum on Specification and Design Languages, 2006.

32. E. Riccobene, P. Scandurra, A. Rosti, and S. Bocchio. A model-driven design environment for embedded systems. In $D A C$ '06: Proceedings of the 43rd annual conference on Design automation, pages 915-918, New York, NY, USA, 2006. ACM Press.

33. E. Riccobene, P. Scandurra, A. Rosti, and S. Bocchio. Designing a unified process for embedded systems. In Fourth International workshop on Model-based Methodologies for Pervasive and Embedded Software (MOMPES'O7). IEEE Press, 2007. 
34. E. Riccobene, P. Scandurra, A. Rosti, and S. Bocchio. A UML2 Profile for SystemC 2.1. STMicroelectronics Technical Report, April, 2007.

35. Douglas C. Schmidt. Guest editor's introduction: Modeldriven engineering. Computer, 39(2):25-31, 2006.

36. B. Selic. A Generic Framework for Modeling Resources with UML. In Proceedings of the 16th Symposium on Integrated Circuits and Systems Design (SBCCI'03), 33:64-69, 2000, IEEE Computer Society.

37. R. De Simone and et al. MARTE: A new Profile RFP for the Modelling and Analysis of Real-time Embedded Systems. In UML for SoC Design workshop at DAC'05.

38. OMG. SPEM, formal/08-04-01, 2008.

39. OMG, SysML, Version 1.0, formal/2007-09-01. http://www . omgsysml.org/, 2007.

40. T. Gröetker and S. Liao and G. Martin and S. Swan. System Design with System C. Kluwer Academic Publisher, 2002.

41. T.Moore, Y.Vanderperren and G.Sonck and P.van Oostende and M. Pauwels and W. Dehaene. A Design Methodology for the Development of a Complex System-On-Chip using UML and Executable System Models. In Forum on Specification and Design Languages, ECSL, 2002.

42. Fujitsu Limited, IBM, NEC. A UML Extension for SoC. Draft RFC to OMG, 2005-01-01, 2005.

43. Y. Vanderperren and W. Dehaene. A Model-Driven Development Process for Low Power SoC Using UML. UML for SOC Design, 2005.

44. Y. Vanderperren, M. Pauwels, W. Dehaene, A.Berna, and F. Ozdemir. A SystemC Based System On Chip Modelling and Design Methodology. System $C$ : Methodologies and Applications, 2003.

45. A. Sangiovanni Vincentelli. Defining Platform-based Design. EEDesign, February 2002.

46. Q. Zhu, A. Matsuda, S. Kuwamura, T. Nakata, and M. Shoji. An object-oriented design process for system-onchip using UML. In Proc. of the 15th international symposium on System Synthesis, Kyoto, Japan, 2002. 